PROCEEDINGS OF THE

AMERICAN MATHEMATICAL SOCIETY

Volume 131, Number 7, Pages 2129-2131

S 0002-9939(02)06849-1

Article electronically published on December 30, 2002

\title{
ALGEBRAIC STRUCTURES DETERMINED BY 3 BY 3 MATRIX GEOMETRY
}

\author{
MARTIN E. WALTER \\ (Communicated by David R. Larson) \\ Dedicated to Masamichi and Kyoko Takesaki and the memory of Yuki

\begin{abstract}
Using a "3 by 3 matrix trick" we show that multiplication (an algebraic structure) in a $C^{*}$-algebra $\mathcal{A}$ is determined by the geometry of the $C^{*}$-algebra of the 3 by 3 matrices with entries from $\mathcal{A}, M_{3}(\mathcal{A})$. This is an example of an algebra-geometry duality which, we claim, has applications.
\end{abstract}

\section{INTRODUCTION}

The motivation for this research was the question, posed about 25 years ago in [10, to find a "natural" generalization of the van Kampen-Pontriagin duality theorem to any locally compact group $G$, based on the order structure of the semigroup of continuous positive definite functions on $G, P(G)$. The nonabelian group duality we seek follows in part from a more general duality between the algebraic structure of a $C^{*}$-algebra and its intrinsic matrix geometry. Since the proof of this algebra-geometry duality for $C^{*}$-algebras is relatively simple and of independent interest, we present the result in this note.

\section{KEY LEMMA}

In 8 J. L. Smul'jan proved a version of our key lemma which was subsequently translated from Russian to English (and also into contemporary operator-theoretic language) by the late Domingo Herrero. A slight modification of our key lemma, more appropriate to numerical linear algebra because it does not involve taking the square root of operators, can be found in [1]. The version of our key lemma which we state below can be found in [2].

In the following lemma $\mathcal{H}_{1}, \mathcal{H}_{2}$ are Hilbert spaces, $\mathcal{B}\left(\mathcal{H}_{i}, \mathcal{H}_{j}\right)$ is the set of all bounded linear operators from $\mathcal{H}_{i}$ to $\mathcal{H}_{j}, i=1,2, j=1,2$, and $A \in \mathcal{B}\left(\mathcal{H}_{1}, \mathcal{H}_{1}\right)$, $B \in \mathcal{B}\left(\mathcal{H}_{2}, \mathcal{H}_{1}\right), B^{*} \in \mathcal{B}\left(\mathcal{H}_{1}, \mathcal{H}_{2}\right), D \in \mathcal{B}\left(\mathcal{H}_{2}, \mathcal{H}_{2}\right)$. Double vertical bars, $\|\cdot\|$, denote the usual uniform (sup) operator norm, e.g., $\|D\|=\sup \left\{\|D \zeta\|_{\mathcal{H}_{2}} \mid\|\zeta\|_{\mathcal{H}_{2}} \leq 1\right\}$.

Key lemma. Let $\widetilde{A}=\left[\begin{array}{cc}A & B \\ B^{*} & D\end{array}\right]$. If $\widetilde{A}$ is a positive operator in $\mathcal{H}_{1} \oplus \mathcal{H}_{2}$, then there exists a bounded operator $X \in \mathcal{B}\left(\mathcal{H}_{2}, \mathcal{H}_{1}\right)$ satisfying the following conditions.

Received by the editors July 15, 2001 and, in revised form, February 10, 2002.

2000 Mathematics Subject Classification. Primary 46L89, 43A35; Secondary 43A40, 43A30.

Key words and phrases. $C^{*}$-algebra, convolution, completely bounded, duality, FourierStieltjes algebra, locally compact group, positive definite function, matrix entry, unitary representation. 
(1) $B=A^{1 / 2} X$,

(2) Range $(X) \subseteq \overline{\operatorname{Range}(A)}$.

On the other hand, if $B$ is of the above form in (1) for some operator $X$, then $\widetilde{A} \geq 0$ if and only if $D \geq X^{*} X$.

\section{The DUALity BETWEen THE PRODUCT OPERATION in $C^{*}$-Algebra $\mathcal{A}$ and the geOmetry in $M_{3}(\mathcal{A})$}

In the following $M_{3}(\mathcal{A})$ is the set of 3 by 3 matrices with entries from $C^{*}$ algebra $\mathcal{A}$. With the usual operations of matrix addition, multiplication and taking the adjoint, $M_{3}(\mathcal{A})$ is a $C^{*}$-algebra; cf. [9], p. 192. The definition of "the geometry of $M_{3}(\mathcal{A})$ " will be evident in the subsequent discussion.

Theorem 1. If $a, b \in \mathcal{A}, a C^{*}$-algebra with unit $e$, then the product $a b$ in $\mathcal{A}$ is determined by the geometry of $M_{3}(\mathcal{A})$, most notably the order structure of $M_{3}(\mathcal{A})$.

The proof of Theorem 1 depends on the following lemma.

Lemma 1. Let $\mathcal{A}$ be a $C^{*}$-algebra with unit e. Let $a, b$ and $c$ be unitary operators in $\mathcal{A}$, e.g., $a^{*} a=e=a a^{*}$. We have

$$
\left(\begin{array}{ccc}
e & a & b \\
a^{*} & e & c \\
b^{*} & c^{*} & e
\end{array}\right) \geq 0 \quad \text { in } \quad M_{3}(\mathcal{A})
$$

if and only if $c=a^{-1} b$ if and only if $b=a c$.

Proof of Lemma 1. In the notation of our key lemma above, $\widetilde{A}$ is the 3 by 3 matrix given in the statement of Lemma 1 , and $A=\left(\begin{array}{cc}e & a \\ a^{*} & e\end{array}\right)$. Since $a$ is unitary, $A^{1 / 2}=\frac{1}{\sqrt{2}} A$. We assume $\widetilde{A}$ is a positive operator in the $C^{*}$-algebra $M_{3}(\mathcal{A})$, hence it will be a positive operator in any realization of $M_{3}(\mathcal{A})$ on Hilbert space.

Thus by (1) of our key lemma, there will be bounded operators $x_{1}, x_{2}$ satisfying the following pair of equations:

$$
\begin{aligned}
e x_{1}+a x_{2} & =\sqrt{2} b, \\
a^{*} x_{1}+e x_{2} & =\sqrt{2} c .
\end{aligned}
$$

Multiplying the first equation by $a^{*}=a^{-1}$ immediately yields $c=a^{-1} b$.

The converse is elementary, since $\left(\begin{array}{lll}e & a & b \\ 0 & 0 & 0 \\ 0 & 0 & 0\end{array}\right)^{*}\left(\begin{array}{ccc}e & a & b \\ 0 & 0 & 0 \\ 0 & 0 & 0\end{array}\right) \geq 0$; cf. [9], p. 193.

Proof of Theorem 1. We consider the order structure on $M_{3}(\mathcal{A})$ to be part of "geometry". Furthermore, note that $\frac{1}{2} A=\frac{1}{2}\left(\begin{array}{c}e^{e} \\ a^{*} \\ e\end{array}\right) \in M_{2}(\mathcal{A})$ is a projection in $C^{*}$ algebra $M_{2}(\mathcal{A})$, i.e., $\left(\frac{1}{2} A\right)^{*}=\frac{1}{2} A=\left(\frac{1}{2} A\right)^{2}$, if and only if $a$ is a unitary in $C^{*}$-algebra $\mathcal{A}$. Projections in a $C^{*}$-algebra are characterized geometrically as the extreme points of the convex intersection of the unit ball and the positive cone of the $C^{*}$ algebra; cf. 7], p. 484. Similarly projections $\frac{1}{2}\left(\begin{array}{c}e \\ b^{*} \\ e\end{array}\right)$ and $\frac{1}{2}\left(\begin{array}{cc}e & c \\ c^{*} & e\end{array}\right)$ determine (and are determined by) unitaries $b$ and $c$, respectively. Since every element in a $C^{*}$ algebra with unit is a linear combination of unitaries (cf. 4]), we have determined the product in $\mathcal{A}$ by various aspects of the geometry of $\mathcal{A}, M_{2}(\mathcal{A})$ and $M_{3}(\mathcal{A})$, and all three of these $C^{*}$-algebras can be considered subalgebras of $M_{3}(\mathcal{A})$. 


\section{Possible APPLICATIONS}

In [12, which is in preparation, we apply Theorem 1 above to construct the duality for locally compact groups mentioned in the Introduction as our motivation for searching for Theorem 1. It is our as yet unrealized goal to make a significant contribution to (via a simplification of) the existing classification of finite groups using our duality theory.

In the "standard model" which appears in theoretical physics, 3 by 3 matrices play a role. This was brought to my attention by a lecture of Alain Connes. Whether the structures explored in this paper might contribute any insights in physics, however minor, remain to be discovered.

\section{ACKNOWLEDGMENTS}

I thank Professor Paul Muhly at the University of Iowa not only for connecting me with the work of Smul'jan and Curto but also for financially supporting a full week visit to the University of Iowa where key points of this work were accomplished.

I would also like to mention that I have profitably discussed this work and/or possible applications at the Great Plains Operator Theory Symposium with Professors R. Curto, B. Blackadar, A. Kumjian, V. Paulsen, J. Quigg and J. Spielberg. A hearty thanks also goes to the referee who pointed out misprints and a missing hypothesis in the statement of Lemma 1 which is indeed used in its proof.

\section{REFERENCES}

1. R. E. Curto and L. A. Fialkow, Solution of the truncated complex moment problem for flat data, Memoirs of the American Mathematical Society, No. 568, Providence, RI, 1996. MR 96g:47009

2. K. R. Davidson, Nest Algebras: triangular forms for operator algebras on Hilbert space, Pitman research notes in mathematics series, No. 191, Harlow, Essex, England: Longman Scientific \& Technical, New York, 1988. MR 90f:47062

3. J. Dixmier, Les $C^{*}$-Algébres et leurs Représentations, Cahiers Scientifiques, Fasc. 29, Gauthier-Villars, Paris 1964. MR 30:1404

4. J. Dixmier, Les algébres d'opérateurs dans l'space hilbertien, Cahiers Scientifiques, Fasc. 25, Gauthier-Villars, Paris, 1969. MR 50:5482

5. E. Hewitt and K. A. Ross, Abstract harmonic analysis, Vol. 1, Springer-Verlag, Berlin, 1963. MR 28:158

6. E. Hewitt and K. A. Ross, Abstract harmonic analysis, Vol. 2, Springer-Verlag, Berlin, New York, 1970. MR 41:7378

7. R. V. Kadison and J. R. Ringrose, Fundamentals of the Theory of Operator Algebras: Advanced Theory, Vol. 2, American Mathematical Society Graduate Studies in Mathematics, Vol. 16, Providence, Rhode Island, 1997. MR 98f:46001b

8. J. L. Smul'jan, An operator Hellinger integral, Mat. Sb. 91 (1959), 381-430. (Russian) MR 22:12396

9. M. Takesaki, Theory of operator algebras, Vol. 1, Springer-Verlag, New York, 1979. MR 81e:46038

10. M. E. Walter, Duality theory for nonabelian locally compact groups, Symposia Mathematica, XXII (1977), 47-59. MR 58:6059

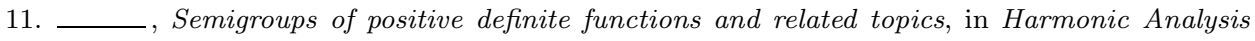
and Hypergroups, (eds: K. A. Ross and A. Schwartz) Birkhäuser, Boston, Mass., 1995, pp.215226. MR 99c:43007

12. _ An algebra-geometry duality, (provisional title) in preparation.

Department of Mathematics, Campus Box 395, University of Colorado, Boulder, Colorado 80309

E-mail address: walter@euclid.colorado.edu 